\title{
ON THE THEORY OF INTERNAL WAVES OF PERMANENT FORM IN FLUIDS OF GREAT DEPTH
}

\author{
C. J. AMICK $\dagger$
}

\section{INTRODUCTION}

This paper concerns the existence of solitary waves in a two-layer perfect fluid of infinite depth contained above a rigid horizontal bottom. In an important paper [1] Benjamin derived a model equation for the position $u$ of the interface between the two fluids:

$$
H\left(u^{\prime}\right)(x)=u(x)-u(x)^{2},
$$

where $H$ denotes the Hilbert transform

$$
H(w)(x)=\frac{1}{\pi} \int_{-\infty}^{\infty} \frac{w(s)}{s-x} d s, \quad x \in \mathbb{R},
$$

defined for suitable functions $w$. In (1.1) certain dimensionless constants have been normalized to unity. Equation (1.1) was also derived by Davis and Acrivos [2] in an experimental and numerical study of travelling waves. A timedependent version of (1.1) was derived by Ono [3] using nonlinear perturbation methods with the full Euler equations.

The fluid is in a semi-infinite domain

$$
\Omega=\{(x, y): x \in \mathbb{R}, y>-h\}
$$

where $h>0$ is a given parameter. Upon scaling, we may take $h=1$, whence $\Omega=\mathbb{R} \times(-1, \infty)$. The fluid domain $\Omega$ consists of a lower fluid region $\Omega_{-}$ in which the density may be normalized to equal unity, an upper region $\Omega_{+}$in which the density is a given constant $\rho \in(0,1)$, and the unknown interface $\Gamma$ between these two regions: $\Gamma=\{(x, Z(x)): x \in \mathbb{R}\}$. Here $Z$ is a smooth function with $Z( \pm \infty)=0$.

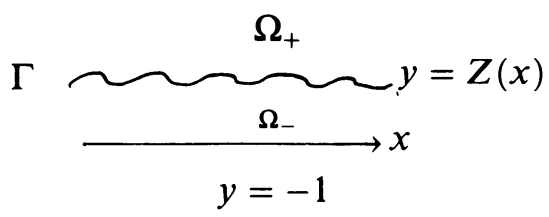

We introduce a 'pseudo stream function' $\psi$ as follows. Let $q$ denote the velocity field in $\Omega_{-} \cup \Omega_{+}$, and define $\psi$ by $q=\left(\psi_{y},-\psi_{x}\right)$ in $\Omega_{-}$and $\sqrt{\rho} q=$

Received by the editors December 5, 1990.

1980 Mathematics Subject Classification (1985 Revision). Primary 76B25; Secondary 76V05.

$\dagger$ This paper is being published posthumously. Charles Amick died on June 3, 1991. 
$\left(\psi_{y},-\psi_{x}\right)$ in $\Omega_{+}$. The interface $\Gamma$ is assumed to be a streamline, whence $\psi=$ 0 on $\Gamma$ without loss of generality. We write $\psi_{+}$and $\psi_{-}$for the restrictions of $\psi$ to $\Omega_{+}$and $\Omega_{-}$. Since $q$ is supposed to approach some constant vector $(c, 0)$ at infinity in $\Omega$, it is natural to define

$$
\Psi(y)= \begin{cases}c y, & y \leq 0 \\ c \sqrt{\rho} y, & y \geq 0\end{cases}
$$

and demand that $\psi(x, y) \rightarrow \Psi(y)$ at infinity in $\Omega$, where the sense of convergence will be made precise later. The constant $c$ is unknown and plays the role of a bifurcation parameter.

Since the fluid is perfect, the quantity $\left(\psi_{y},-\psi_{x}\right)$ is irrotational and this implies that $\psi_{ \pm}$is harmonic in $\Omega_{ \pm}$. Bernoulli's equation and the demand that the pressure be continuous across $\Gamma$ yield

$$
\frac{1}{2}\left\{\left|\nabla \psi_{+}\right|^{2}-\left|\nabla \psi_{-}\right|^{2}\right\}=(\rho-1)\left(c^{2} / 2-g y\right) \text { on } \Gamma
$$

where $g$ is the gravitational constant and $\left|\nabla \psi_{ \pm}\right|$denotes the limiting value on $\Gamma$ as taken from $\Omega_{ \pm}$. The mathematical problem may be summarized as one of finding a constant $c>0$, a function $Z$ defining an interface $\Gamma$, and functions $\psi_{ \pm}$satisfying

$$
\begin{gathered}
\Delta \psi_{ \pm}=0 \text { in } \Omega_{ \pm}, \\
\psi_{+}=\psi_{-}=0 \text { on } \Gamma, \\
\psi_{-}(x,-1)=\Psi(-1)=-c, \quad x \in \mathbb{R}, \\
\psi \rightarrow \Psi \text { at infinity in } \Omega, \\
\frac{1}{2}\left\{\left|\nabla \psi_{+}\right|^{2}-\left|\nabla \psi_{-}\right|^{2}\right\}=(\rho-1)\left(c^{2} / 2-g y\right) \text { on } \Gamma .
\end{gathered}
$$

For any constant $c$ there is the trivial solution $Z \equiv 0$ and $\psi \equiv \Psi$; our interest lies with nontrivial solutions.

We note that if $\rho$ is set to zero and $\psi_{+} \equiv 0$, then (1.3)-(1.7) is the usual problem for 'water waves' $[4,5]$. For that case, solitary waves decay exponentially at infinity whereas for the case $\rho>0$, we shall show that $Z(x)=O\left(x^{-2}\right)$.

The paper is organized as follows. In $\S 2$ we introduce some notation and function spaces. Section 3 considers (1.3) in $\Omega_{+}$with (1.4), and there is derived a (nonlocal) representation for $\left|\nabla \psi_{+}\right|$on $\Gamma$ in terms of the function $Z$ defining $\Gamma$. In $\S 4$ we perform a hodograph transform on the unknown domain $\Omega_{-}$, and arrive at an equivalent problem of solving Laplace's equation in a fixed strip $\mathbb{R} \times(-1,0)$ with Dirichlet boundary data on the bottom and a nonlinear, nonlocal boundary condition, derived from (1.7), on the top. The analysis of the problem is performed in $\S 5$, and after suitable scaling with a small parameter, the problem is reduced to one of the form (1.1) with an additional small correction term. This final equation is analyzed with the aid of the implicit function theorem, and there is shown to exist (Theorem 8) a branch of small solutions with (cf. (5.13))

$$
Z(x)=\varepsilon \widetilde{Z}(\varepsilon x) \simeq \frac{2}{3}(1-\rho) \varepsilon \widetilde{Z}_{0}\left(\frac{1-\rho}{\rho} \varepsilon x\right),
$$

where $\varepsilon>0$ is small and is related to $c$ by the relation $\varepsilon=g c_{0}^{-2}-g c^{-2}$. Here $c_{0}=\sqrt{(1-\rho) g}$ is the critical speed at which the bifurcation of small solutions 
occurs, and

$$
\tilde{Z}_{0}(x)=\frac{2}{1+x^{2}} .
$$

(It is known [6, 7] that $\widetilde{Z}_{0}$ is the unique, modulo translation, solitary wave solution of (1.1).)

\section{Notation}

Let

$$
T_{+}=\{(x, y): x \in \mathbb{R}, y>0\},
$$

and

$$
T_{-}=\{(x, y): x \in \mathbb{R},-1<y<0\} .
$$

For a set $S$ in $\mathbb{R}$ or $\mathbb{R}^{2}$ and a function $u$ defined on $S$, define for $p \in S$

$$
[u](p)=|u(p)|+\sup _{\substack{\tilde{p} \in S \\|p-\tilde{p}|<1}} \frac{|u(p)-u(\tilde{p})|}{|p-\tilde{p}|^{\alpha}}
$$

where $\alpha \in(0,1)$ is given and fixed. Define the following function spaces:

$$
A=\left\{u \in C^{1+\alpha}\left(\bar{T}_{+}\right): \sup _{(x, y) \in T_{+}}\left(1+r^{2}\right)\left\{\frac{|u(x, y)|}{2+y}+[\nabla u](x, y)\right\}=|u|_{A}<\infty\right\}
$$

where $r^{2}=x^{2}+y^{2}$;

$$
\begin{aligned}
& B=\left\{u \in C^{\alpha}\left(\bar{T}_{+}\right): \sup _{(x, y) \in T_{+}}\left(1+r^{4}\right)[u](x, y)=|u|_{B}<\infty\right\} ; \\
& D=\left\{u \in C^{1+\alpha}(\mathbb{R}): \sup _{x \in \mathbb{R}}\left(1+x^{2}\right)\left\{|u(x)|+\left[u^{\prime}\right](x)\right\}=|u|_{D}<\infty\right\} ; \\
& E=\left\{u \in C^{\alpha}(\mathbb{R}): \sup _{x \in \mathbb{R}}\left(1+x^{2}\right)[u](x)=|u|_{E}<\infty\right\} ; \\
& F=\left\{u \in C^{1+\alpha}\left(\bar{T}_{-}\right): \sup _{(x, y) \in T_{-}}\left(1+x^{2}\right)\{|u(x, y)|+[\nabla u](x, y)\}=|u|_{F}<\infty\right\} .
\end{aligned}
$$

\section{Estimates IN $\Omega_{+}$}

In this section we consider the problem

$$
\begin{aligned}
\Delta \psi_{+}=0 & \text { in } \Omega_{+}=\{(x, y): y>Z(x), x \in \mathbb{R}\}, \\
\psi_{+}=0 & \text { on } \Gamma=\{(x, Z(x)): x \in \mathbb{R}\}, \\
\psi_{+} \rightarrow \Psi & \text { at infinity in } \Omega_{+},
\end{aligned}
$$

with the aim of estimating $\left|\nabla \psi_{+}\right|$on $\Gamma$ in terms of the function $Z$. If $Z \equiv 0$, we have the trivial solution $\psi_{+} \equiv \Psi$. Since we are interested in solutions with $\psi_{+}$close to $\Psi$, we shall assume that

$$
\frac{\partial}{\partial y} \psi_{+}>0 \text { in } \Omega_{+} .
$$

This allows us to solve for $y$ as a function of $(x, \psi), y=y(x, \psi)$, say. We continue to follow the technique in $[8,9]$ and define

$$
w\left(x_{1}, x_{2}\right)=y\left(x_{1}, \Psi\left(x_{2}\right)\right)-x_{2}, \quad\left(x_{1}, x_{2}\right) \in T_{+} .
$$


The use of this in (3.1)-(3.3) yields

$$
\begin{gathered}
\frac{\partial}{\partial x_{1}} f_{1}(\nabla w)+\frac{\partial}{\partial x_{2}} f_{2}(\nabla w)=0 \text { in } T_{+}, \\
w \rightarrow 0 \text { at infinity in } T_{+}, \\
w\left(x_{1}, 0\right)=Z\left(x_{1}\right), \quad x_{1} \in \mathbb{R} .
\end{gathered}
$$

Here $f\left(p_{1}, p_{2}\right)=\left(p_{1}^{2}+p_{2}^{2}\right) /\left(2+2 p_{2}\right)$ and $f_{i}=\partial f / \partial p_{i}, i=1,2$.

It is elementary to check that

$$
\left|\nabla \psi_{+}\left(x_{1}, Z\left(x_{1}\right)\right)\right|^{2}=c^{2} \rho \frac{\left(1+w_{x_{1}}\left(x_{1}, 0\right)^{2}\right)}{\left(1+w_{x_{2}}\left(x_{1}, 0\right)\right)^{2}}, \quad x_{1} \in \mathbb{R} .
$$

Since $w_{x_{1}}\left(x_{1}, 0\right)=Z^{\prime}\left(x_{1}\right)$, we need only find $w_{x_{2}}$. Equation (3.5) may be rewritten as

$$
\Delta w=\operatorname{div}\left(g_{1}, g_{2}\right) \text { in } T_{+},
$$

where

$$
g_{1}=\frac{w_{x_{1}} w_{x_{2}}}{1+w_{x_{2}}}, \quad g_{2}=\frac{w_{x_{2}}^{2}}{1+w_{x_{2}}}+\frac{|\nabla w|^{2}}{2\left(1+w_{x_{2}}\right)^{2}} .
$$

For small $w,\left(g_{1}, g_{2}\right)$ is quadratically small, whence $w$ should be approximated well by the solution $W$ of Laplace's equation with the boundary conditions (3.6)-(3.7). More precisely, we set $w=W+R$, where

$$
W\left(x_{1}, x_{2}\right)=L(Z)\left(x_{1}, x_{2}\right) \equiv \frac{x_{2}}{\pi} \int_{-\infty}^{\infty} \frac{Z(t)}{\left(x_{1}-t\right)^{2}+x_{2}^{2}} d t .
$$

We assume that $Z$ belongs to the space $D$ introduced in $\S 2$.

The function $R$ satisfies $\Delta R=\operatorname{div}\left(g_{1}, g_{2}\right)$ in $T_{+}$and $R\left(x_{1}, 0\right)=0, x_{1} \in$ $\mathbb{R}$. This may be solved by the reflection method:

$$
R\left(x_{1}, x_{2}\right)=\Gamma\left(g_{1}, g_{2}\right)\left(x_{1}, x_{2}\right), \quad\left(x_{1}, x_{2}\right) \in T_{+},
$$

where the linear operator $\Gamma$ is defined on $B \times B$ by the formula

$$
\begin{aligned}
\Gamma\left(h_{1}, h_{2}\right)\left(x_{1}, x_{2}\right) \\
=\frac{1}{\pi} \int\left(s-x_{1}\right)\left\{\frac{1}{\left(s-x_{1}\right)^{2}+\left(t-x_{2}\right)^{2}}-\frac{1}{\left(s-x_{1}\right)^{2}+\left(t+x_{2}\right)^{2}}\right\} g_{1} d s d t \\
\quad+\frac{1}{\pi} \int\left\{\frac{t-x_{2}}{\left(s-x_{1}\right)^{2}+\left(t-x_{2}\right)^{2}}-\frac{t+x_{2}}{\left(s-x_{1}\right)^{2}+\left(t+x_{2}\right)^{2}}\right\} g_{2} d s d t
\end{aligned}
$$

for $\left(x_{1}, x_{2}\right) \in T_{+}$and the integral is over $T_{+}$. Equation (3.10) shows that $\left(g_{1}, g_{2}\right)$ depends on $\nabla w$, and the use of $w=W+R$ in (3.12) then yields a nonlinear equation for $R$ depending on $Z$ :

$$
R\left(x_{1}, x_{2}\right)=N(R, Z)\left(x_{1}, x_{2}\right) .
$$

Our goal is to solve this equation for $R$ as a function of $Z$ and to show that $R$ is small, in a suitable sense, compared to $Z$. We begin with a few lemmas. 
Lemma 1. The linear map $L$, defined by (3.11), is a bounded linear map from $D$ to $A$.

Proof. If $Z \in D$, then

$$
\left|W\left(x_{1}, 0\right)\right|=\left|Z\left(x_{1}\right)\right| \leq \frac{|Z|_{D}}{1+x_{1}^{2}} .
$$

The function

$$
\frac{\left(1+x_{2}\right)|Z|_{D}}{\left(1+x_{2}\right)^{2}+x_{1}^{2}}
$$

is harmonic in $T_{+}$and exceeds $|W|$ when $x_{2}=0$, whence by the maximum principle

$$
\left|W\left(x_{1}, x_{2}\right)\right| \leq \frac{\left(1+x_{2}\right)}{\left(1+x_{2}\right)^{2}+x_{1}^{2}}|Z|_{D} .
$$

Fix a point $\left(\tilde{x}_{1}, \tilde{x}_{2}\right), \tilde{x}_{2} \geq 1$, and let $S=\left\{\left(x_{1}, x_{2}\right):\left|\left(x_{1}, x_{2}\right)-\left(\tilde{x}_{1}, \tilde{x}_{2}\right)\right|<\right.$ $\left.\tilde{x}_{2} / 2\right\}$. Then $[10$, p. 90$]$ gives

$$
\left|\nabla W\left(\tilde{x}_{1}, \tilde{x}_{2}\right)\right| \leq \frac{\text { const. }}{\tilde{x}_{2}} \max _{\left(x_{1}, x_{2}\right) \in S}\left|W\left(x_{1}, x_{2}\right)\right| \leq \frac{\text { const. }|Z|_{D}}{1+\tilde{x}_{1}^{2}+\tilde{x}_{2}^{2}} .
$$

Analogous estimates hold for higher derivatives, so

$$
[\nabla W]\left(\tilde{x}_{1}, \tilde{x}_{2}\right) \leq \frac{\text { const. }|Z|_{D}}{1+\tilde{x}_{1}^{2}+\tilde{x}_{2}^{2}} \quad \text { if } \tilde{x}_{2} \geq 1
$$

Elliptic estimates at the boundary give

$$
\begin{aligned}
& |W|_{C^{1+\alpha}\left(\left[x_{1}-1, x_{1}+1\right] \times[0,1]\right)} \\
& \quad \leq \text { const. }\left\{|W|_{L_{\infty}\left(\left[x_{1}-2, x_{1}+2\right] \times[0,2]\right)}+|Z|_{C^{1+\alpha}\left(\left[x_{1}-2, x_{1}+2\right]\right)}\right\} \\
& \quad \leq \frac{\text { const. }|Z|_{D}}{1+x_{1}^{2}} . \square
\end{aligned}
$$

Lemma 2. The map $(R, Z) \mapsto\left(g_{1}(\nabla W+\nabla R), g_{2}(\nabla W+\nabla R)\right)$ is a smooth map from a sufficiently small neighborhood of $(0,0)$ in $A \times D$ into $B \times B$.

Proof. With the aid of Lemma 1, we have

$$
\left(1+r^{2}\right)\left\{[\nabla W]\left(x_{1}, x_{2}\right)+[\nabla R]\left(x_{1}, x_{2}\right)\right\} \leq \text { const. }\left(|Z|_{D}+|R|_{A}\right)
$$

and the result is immediate.

In order to apply the implicit function theorem to (3.12) or (3.14), we need the following

Lemma 3. The linear map $\Gamma$, defined by (3.13), is bounded from $B \times B$ into $A$.

Proof. The proof is technical and will be given in the Appendix.

The preceding lemmas together ensure that the map $(R, Z) \mapsto N(R, Z)$ is smooth on a neighborhood of $(0,0)$ in $A \times D$ into $A$, and clearly $N_{R}(0,0)=$ 0 . 
Theorem 4. There exists a neighborhood $U$ of 0 in $D$, a neighborhood $V$ of $(0,0)$ in $A \times D$ and a smooth map $\mu: U \rightarrow A$ such that $\{(\mu(Z), Z): Z \in U\}$ consists of all solutions of $R=N(R, Z)$ in $V$. Moreover,

$$
|\mu(Z)|_{A} \leq \text { const. }|Z|_{D}^{2} .
$$

Proof. The first part of the theorem is immediate from the implicit function theorem. The estimate (3.16) is due to the fact that $N_{Z}(0,0)=0$.

Remark 1. In our main result (1.8) we seek $Z(x)=\varepsilon \widetilde{Z}(\varepsilon x)$, and it is natural to see what effect this has on the result of Theorem 4 . More precisely, fix $\widetilde{Z}_{1} \in D$ and for each $\tilde{Z}$ in a neighborhood of $\widetilde{Z}_{1}$ define $Z\left(x_{1}\right)=\varepsilon \widetilde{Z}\left(\varepsilon x_{1}\right)$ for small $\varepsilon>0$. Note from (3.11) that

$$
(L Z)\left(\frac{x_{1}}{\varepsilon}, \frac{x_{2}}{\varepsilon}\right)=\varepsilon L(\tilde{Z})\left(x_{1}, x_{2}\right), \quad\left(x_{1}, x_{2}\right) \in T_{+},
$$

and that

$$
\begin{aligned}
\left(\frac{\partial}{\partial x_{2}} L Z\right)\left(\frac{x_{1}}{\varepsilon}, 0\right) & =\varepsilon^{2}\left(\frac{\partial}{\partial x_{2}} L \tilde{Z}\right)\left(x_{1}, 0\right)=\frac{\varepsilon^{2}}{\pi} \int_{-\infty}^{\infty} \frac{\tilde{Z}^{\prime}(t)}{t-x_{1}} d t \\
& =\varepsilon^{2} H\left(\tilde{Z}^{\prime}\right)\left(x_{1}\right)
\end{aligned}
$$

where $H$ denotes the Hilbert transform (cf. (1.2)). Given $\widetilde{Z}$ and defining $Z$ as above, we obtain a function $\mu(Z)$ from Theorem 4 and may then define

$$
\tilde{\mu}(\tilde{Z})\left(x_{1}, x_{2}\right)=\varepsilon^{-1} \mu(Z)\left(\frac{x_{1}}{\varepsilon}, \frac{x_{2}}{\varepsilon}\right) .
$$

It is elementary to conclude from what has come before that

$$
|\tilde{\mu}(\widetilde{Z})|_{A} \leq \text { const. } \varepsilon^{2}|\widetilde{Z}|_{D}^{2} .
$$

Theorem 4 shows that (3.5)-(3.7) has a unique solution for each sufficiently small $Z \in D$. In particular, the quantity $\left|\nabla \psi_{+}\left(x_{1}, Z\left(x_{1}\right)\right)\right|$ is determined by (3.8). If we fix $\widetilde{Z} \in D$ and define $Z\left(x_{1}\right)=\varepsilon \widetilde{Z}\left(\varepsilon x_{1}\right)$ for $\varepsilon>0$, then (3.8) yields

$$
\begin{aligned}
\left|\nabla \psi_{+}\left(\frac{x_{1}}{\varepsilon}, \varepsilon \tilde{Z}\left(x_{1}\right)\right)\right|^{2} & =\frac{c^{2} \rho\left\{1+\varepsilon^{4} \widetilde{Z}^{\prime}\left(x_{1}\right)^{2}\right\}}{\left\{1+\varepsilon^{2} H\left(\widetilde{Z}^{\prime}\right)\left(x_{1}\right)+O\left(\varepsilon^{4}|\widetilde{Z}|_{D}^{2}\right)\right\}^{2}} \\
& =c^{2} \rho\left(1-2 \varepsilon^{2} H\left(\widetilde{Z}^{\prime}\right)\left(x_{1}\right)+\Sigma(\widetilde{Z} ; \varepsilon)\left(x_{1}\right)\right) .
\end{aligned}
$$

Here $\Sigma$ is the remainder term and is estimated by

$$
|\Sigma(\tilde{Z} ; \varepsilon)|_{E} \leq \text { const. } \varepsilon^{4}|\widetilde{Z}|_{D}^{2} .
$$

We shall use the formula (3.20) in the nonlinear boundary condition (1.7).

\section{REDUCTION TO A PROBLEM IN A STRIP}

In this section we consider (1.3)-(1.7) for the function $\psi_{-}$when the term $\left|\nabla \psi_{+}\right|$in (1.7) is related to the function $Z$, defining $\Gamma$, via (3.20). In order to transform the problem to a fixed domain, one might proceed as in $\S 3$ and derive (3.5)-(3.7) (now holding in $T_{-}$) together with the relevant version of (1.7) to hold on $\left\{x_{2}=0\right\}$. We would like to solve (3.9) as explicitly as possible, use the results in the nonlinear nonlocal boundary condition on $\left\{x_{2}=0\right\}$, and then perform the type of scaling $\left(x_{1} \rightarrow \varepsilon x_{1}\right)$ discussed at the end of $\S 3$. Rather than 
pursue this course, we have decided to use a hodograph transformation since the resulting partial differential equation will be Laplace's rather than (3.9).

For a point $z \in \Omega_{-}$we write either $z=x+i y$ or $z=(x, y)$. Define

$$
P(z)=P_{1}(z)+i P_{2}(z)=\frac{\phi(z)+i \psi_{-}(z)}{c}, \quad z \in \Omega_{-},
$$

where $\phi$ denotes a harmonic conjugate to $\psi_{-}$. Note that $P_{2}(x,-1)=-1$ and $P_{2}=0$ on $\Gamma$ by (1.4)-(1.5). We assume that $P$ is an injective mapping of $\bar{\Omega}_{-}$ onto $\bar{T}_{-}=\mathbb{R} \times[-1,0]$; this is easily accomplished by demanding that $\partial \psi_{-} / \partial y$ is bounded away from zero on $\bar{\Omega}_{-}$. Denote points in $\bar{T}_{-}$by $\zeta=\xi+i \eta$ and let $Q$ be the inverse of $P$ so that

$$
x+i y=Q(\zeta)=Q_{1}(\zeta)+i Q_{2}(\zeta), \quad \zeta \in \bar{T}_{-} .
$$

The free surface $\Gamma$ is given by

$$
\left\{\left(Q_{1}(\xi, 0), Q_{2}(\xi, 0): \xi \in \mathbb{R}\right\},\right.
$$

and an easy calculation yields

$$
\left|\nabla \psi_{-}\left(Q_{1}(\xi, 0), Q_{2}(\xi, 0)\right)\right|^{2}=\frac{c^{2}}{\left|Q^{\prime}(\xi, 0)\right|^{2}} .
$$

The use of this in (1.7) gives

together with

$$
\begin{aligned}
\mid \nabla \psi_{+} & \left.\left(Q_{1}(\xi, 0), Q_{2}(\xi, 0)\right)\right|^{2}-\frac{c^{2}}{\left|Q^{\prime}(\xi, 0)\right|^{2}} \\
= & (\rho-1)\left(c^{2}-2 g Q_{2}(\xi, 0)\right), \quad \xi \in \mathbb{R},
\end{aligned}
$$

$$
\begin{gathered}
\Delta Q_{2}=0 \quad \text { in } T_{-}=\mathbb{R} \times(-1,0), \\
Q_{2}(x,-1)=-1, \quad \xi \in \mathbb{R} .
\end{gathered}
$$

Now

$$
\begin{aligned}
& \left|\nabla \psi_{+}\left(Q_{1}(\xi, 0), Q_{2}(\xi, 0)\right)\right|^{2}=c^{2} \rho\left\{1-2 H\left(Z^{\prime}\right)\left(Q_{1}(\xi, 0)\right)\right\} \\
& +\left\{\left|\nabla \psi_{+}\left(Q_{1}(\xi, 0), Q_{2}(\xi, 0)\right)\right|^{2}-c^{2} \rho\left(1-2 H\left(Z^{\prime}\right)\left(Q_{1}(\xi, 0)\right)\right)\right\}
\end{aligned}
$$

where

$$
\begin{aligned}
H\left(Z^{\prime}\right)\left(Q_{1}(\xi, 0)\right) & =\frac{1}{\pi} \int_{-\infty}^{\infty} \frac{Z^{\prime}(t) d t}{t-Q_{1}(\xi, 0)} \\
& =\frac{1}{\pi} \int_{-\infty}^{\infty} \frac{Z^{\prime}\left(Q_{1}(s, 0)\right)\left(Q_{1}\right)_{\xi}(s, 0) d s}{Q_{1}(s, 0)-Q_{1}(\xi, 0)} \\
& =\frac{1}{\pi} \int_{-\infty}^{\infty} \frac{\left(Q_{2}\right)_{\xi}(s, 0) d s}{Q_{1}(s, 0)-Q_{1}(\xi, 0)} .
\end{aligned}
$$

For trivial flow $P$ and $Q$ are the identity maps, and, since we are interested in small solutions, we set $Q_{1}(\xi, \eta)=\xi+S_{1}(\xi, \eta), Q_{2}(\xi, \eta)=\eta+S_{2}(\xi, \eta)$ where $S_{1}$ and $S_{2}$ should be small. If this representation is used in (4.3)-(4.7) and linear terms collected on the left in the boundary conditions, then

$$
\begin{gathered}
\Delta S_{2}=0 \text { in } T_{-} \\
S_{2}(\xi,-1)=0, \quad \xi \in \mathbb{R}, \\
-\frac{\rho}{\pi} \int_{-\infty}^{\infty} \frac{\left(S_{2}\right)_{\xi}(t, 0) d t}{t-\xi}+\left(S_{2}\right)_{\eta}(\xi, 0)-S_{2}(\xi, 0)=f(\xi), \quad \xi \in \mathbb{R},
\end{gathered}
$$


where $f=f_{1}+\cdots+f_{4}$ and

(4.10a)

$$
f_{1}(\xi)=\frac{\rho}{\pi} \int_{-\infty}^{\infty}\left(S_{2}\right)_{\xi}(t, 0)\left\{\frac{1}{t-\xi+S_{1}(t, 0)-S_{1}(\xi, 0)}-\frac{1}{t-\xi}\right\} d t
$$

$$
f_{2}(\xi)=-\left\{\left|\nabla \psi_{+}\left(Q_{1}(\xi, 0), Q_{2}(\xi, 0)\right)\right|^{2}-c^{2} \rho\left(1-2 H\left(Z^{\prime}\right)\left(Q_{1}(\xi, 0)\right)\right)\right\} / 2 c^{2} ;
$$

$$
f_{3}(\xi)=\frac{1}{2}\left\{\frac{1}{\left(1+\left(S_{2}\right)_{\eta}\right)^{2}\left(S_{2}\right)_{\xi}^{2}}-1+2\left(S_{2}\right)_{\eta}\right\},
$$

the right-hand side being evaluated at $(\xi, 0)$;

$$
f_{4}(\xi)=g(1-\rho)\left(c^{-2}-c_{0}^{-2}\right) S_{2}(\xi, 0) .
$$

Here $c_{0} \sqrt{g(1-\rho)}$ is the critical speed at which we expect bifurcation to occur. For $c>0$, we define a bifurcation parameter $\varepsilon \in \mathbb{R}$ by the formula

$$
\varepsilon=\frac{-g}{c^{2}}+\frac{g}{c_{0}^{2}} \text {. }
$$

We shall show the existence of a branch of solutions for small $\varepsilon>0$. Before giving the rigorous results, we show formally how (4.8)-(4.11) lead to (1.1).

4.2 Some formal calculations. Consider $f$ in (4.9) as given; in $\S 4.3$, we shall seek a solution of (4.8)-(4.9) in the form $S_{2}(\xi, \eta)=U(\xi)(1+\eta)+R(\xi, \eta)$ where $-\rho H\left(U^{\prime}\right)(\xi)=f(\xi)$. This will lead to an explicit formula (4.18) for the remainder $R$ in terms of $f$. If $f$ now depends on $U$ and $R$ via (4.10), we shall see that $R$ is small, in some sense, compared to $U$.

In this section, we assume $S_{2}(\xi, \eta) \simeq U(\xi)(1+\eta)$, scale $U(\xi)=\varepsilon \widetilde{U}(\varepsilon \xi)$ for $\varepsilon \neq 0$, use this in (4.9)-(4.10) and keep terms to order $\varepsilon^{2}$ :

$$
-\rho \varepsilon|\varepsilon| H\left(\widetilde{U}^{\prime}\right)(\xi) \simeq \frac{3}{2} \varepsilon^{2} \widetilde{U}(\xi)^{2}-(1-\rho) \varepsilon^{2} \widetilde{U}(\xi) .
$$

We shall show in $\S 4.3$ that the terms $f_{1}$ and $f_{2}$ in (4.10a) and (4.10b), respectively, are at least $O\left(\varepsilon^{3}\right)$, and so contribute nothing to (4.12). The two terms on the right-hand side of $(4.12)$ come from $(4.10 \mathrm{c})-(4.10 \mathrm{~d})$. The equation

$$
-\rho \operatorname{sgn}(\varepsilon) H\left(\widetilde{U}^{\prime}\right)=\frac{3}{2} \widetilde{U}^{2}-(1-\rho) \widetilde{U}
$$

has only the trivial solution when $\varepsilon<0$, [6], and may be scaled to agree with (1.1) when $\varepsilon>0$. Since (1.1) has the unique solution (modulo translation) [6] $u(\xi)=2 /\left(1+\xi^{2}\right)$, the solution to $(4.13)$ is

$$
\tilde{U}_{0}(\xi)=\frac{4}{3}(1-\rho)\left\{1+\left(\frac{1-\rho}{\rho} \xi\right)^{2}\right\}^{-1} .
$$

If the transformations which led from (1.3)-(1.7) to (4.8)-(4.10) are reversed, then (4.14) is seen to imply (1.8).

4.3 Decomposition of $S_{2}$. Since we are interested in solutions which tend to zero at least as fast as $\xi^{-2}$ at infinity in $T_{-}$, we may take the Fourier transform in the $\xi$-direction of $(4.8)-(4.9)$. The result is

$$
\widehat{S}_{2}(k, \eta)=\frac{\eta+1}{\rho|k|} \hat{f}(k)+\widehat{G}(k, \eta) \hat{f}(k)
$$


where

$$
\widehat{G}(k, \eta)=\frac{\sinh k(\eta+1)}{k}\left\{\cosh k-\frac{\sinh k}{k}+\frac{\rho|k|}{k} \sinh k\right\}^{-1}-\frac{\eta+1}{\rho|k|} .
$$

We seek $S_{2}$ of the form $S_{2}(\xi, \eta)=U(\xi)(\eta+1)+R(\xi, \eta)$ where

$$
\widehat{U}(k)=\frac{\hat{f}(k)}{\rho|k|}, \quad \widehat{R}(k, \eta)=\widehat{G}(k, \eta) \hat{f}(k) .
$$

It follows that

$$
\begin{gathered}
H\left(U^{\prime}\right)(\xi)=\frac{1}{\pi} \int_{-\infty}^{\infty} \frac{U^{\prime}(t) d t}{t-\xi}=-\frac{f(\xi)}{\rho}, \quad \xi \in \mathbb{R}, \\
R(\xi, \eta)=\frac{1}{\sqrt{2 \pi}} \int_{-\infty}^{\infty} G(\xi-t, \eta) f(t) d t, \quad(\xi, \eta) \in T_{-} .
\end{gathered}
$$

Since $\widehat{G}$ is given explicitly by (4.16), we may evaluate $G$ by the inverse transform and obtain

Lemma 5. (a) $|G(\xi, \eta)|+\left|G_{\xi}(\xi, \eta)\right| \leq$ const. $(\eta+1) \xi^{-2}$ and $\left|G_{\eta}(\xi, \eta)\right| \leq$ const. $\xi^{-2}$ for $|\xi|>\frac{1}{2}$ and $\eta \in(-1,0)$.

(b) For $|\xi| \leq \frac{1}{2}$ and $\eta \in(-1,0)$

$$
\begin{aligned}
G(\xi, \eta) & =\sqrt{2 / \pi}(1+\eta)\left\{\rho^{-1} \log |\xi|-(1+\rho)^{-1} \log \sqrt{\xi^{2}+\eta^{2}}+O(1)\right\} \\
G_{\xi}(\xi, \eta) & =\sqrt{2 / \pi}(1+\eta)\left\{(\rho \xi)^{-1}-(1+\rho)^{-1} \xi /\left(\xi^{2}+\eta^{2}\right)+O(1)\right\} \\
G_{\eta}(\xi, \eta) & =\sqrt{2 / \pi}\left\{\rho^{-1} \log |\xi|-(1+\rho)^{-1}\left[\eta /\left(\xi^{2}+\eta^{2}\right)+\log \sqrt{\left.\xi^{2}+\eta^{2}\right]}+O(1)\right\}\right.
\end{aligned}
$$

The $O(1)$ terms are Hölder continuous for any exponent less than unity.

(c) The linear map $\mathscr{L}$ defined by

$$
(\mathscr{L} g)(\xi, \eta)=\int_{-\infty}^{\infty} G(\xi-t, \eta) g(t) d t, \quad(\xi, \eta) \in T_{-},
$$

is bounded from $E$ to $F$.

Proof. The proof is technical and is given in the Appendix.

Remark 2. Recall from $\S 2$ that $E$ and $F$ are the spaces $C^{\alpha}(\mathbb{R})$ and $C^{1+\alpha}\left(\bar{T}_{-}\right)$, respectively, with the weight $1+\xi^{2}$.

The function $f$ in (4.10) is a nonlinear function of $S_{1}$ and $S_{2}$, and therefore is a nonlinear (and nonlocal) function of $U$ and $R$. In order to analyze and solve (4.17)-(4.18), we shall shortly seek $U(\xi)=\varepsilon \widetilde{U}(\varepsilon \xi)$ and $R(\xi, \eta)=$ $\varepsilon \widetilde{R}(\varepsilon \xi, \eta)$. This will lead us to consider the right-hand side of (4.18) with $f(t)$ replaced by $\varepsilon \tilde{f}(\varepsilon t)$, and the following lemma will be crucial; the proof appears in the Appendix.

Lemma 6. For $\varepsilon>0$, define the linear map

$$
\left(\mathscr{L}_{\varepsilon} g\right)(\xi, \eta)=\frac{1}{\varepsilon} \int_{-\infty}^{\infty} G\left(\frac{\xi-t}{\varepsilon}, \eta\right) g(t) d t, \quad(\xi, \eta) \in T_{-} .
$$

Then

$$
\begin{aligned}
& \sup _{(\xi, \eta) \in T_{-}}\left(1+\xi^{2}\right)\left\{\left[\mathscr{L}_{\varepsilon} g\right](\xi, \eta)+\left[\frac{\partial}{\partial \eta} \mathscr{L}_{\varepsilon} g\right](\xi, \eta)+\varepsilon\left[\frac{\partial}{\partial \xi} \mathscr{L}_{\varepsilon} g\right](\xi, \eta)\right\} \\
& \quad \leq \text { const. }|g|_{E} .
\end{aligned}
$$


The transformation $U(\xi)=\varepsilon \widetilde{U}(\varepsilon \xi)$ in (4.17) yields

$$
H\left(U^{\prime}\right)(\xi)=\frac{\varepsilon^{2}}{\pi} \int_{-\infty}^{\infty} \frac{\widetilde{U}^{\prime}(\varepsilon t) d t}{t-\xi}=\frac{\varepsilon^{2}}{\pi} \int_{-\infty}^{\infty} \frac{\widetilde{U}^{\prime}(t) d t}{t-\varepsilon \xi}
$$

whence

$$
\rho H\left(U^{\prime}\right)(\xi / \varepsilon)=\varepsilon^{2} \rho H\left(\tilde{U}^{\prime}\right)(\xi)=-f(\xi / \varepsilon) .
$$

If we also set $R(\xi, \eta)=\varepsilon \widetilde{R}(\varepsilon \xi, \eta), f(\xi)=\varepsilon \tilde{f}(\varepsilon \xi), f_{1}(\xi)=\varepsilon \tilde{f}_{1}(\varepsilon \xi)$, etc., then (4.17)-(4.18) yield

$$
\begin{aligned}
& \rho H\left(\widetilde{U}^{\prime}\right)(\xi)-(1-\rho) \widetilde{U}(\xi)+\frac{3}{2} \widetilde{U}(\xi)^{2}=(1-\rho) \widetilde{R}(\xi, 0)-\varepsilon^{-1} \tilde{f}_{1}(\xi) \\
& -\varepsilon^{-1} \tilde{f}_{2}(\xi)-\varepsilon^{-1}\left\{\tilde{f}_{3}(\xi)-\frac{3}{2} \varepsilon(\widetilde{U}(\xi)+\widetilde{R}(\xi, 0))^{2}\right\} \\
& \quad-3 \widetilde{U}(\xi) \widetilde{R}(\xi, 0)-\frac{3}{2} \widetilde{R}(\xi, 0)^{2}
\end{aligned}
$$

$$
\widetilde{R}(\xi, \eta)=\frac{1}{\varepsilon \sqrt{2 \pi}} \int_{-\infty}^{\infty} G\left(\frac{\xi-t}{\varepsilon}, \eta\right) \tilde{f}(t) d t
$$

$$
\begin{aligned}
\sqrt{2 \pi} \widetilde{R}_{\xi}(\xi, \eta)= & -(1-\rho) \int_{-\infty}^{\infty} G\left(\frac{\xi-t}{\varepsilon}, \eta\right)\left(\widetilde{U}^{\prime}(t)+\widetilde{R}_{\xi}(t, 0)\right) d t \\
& +\frac{1}{\varepsilon^{2}} \int_{-\infty}^{\infty} G_{\xi}\left(\frac{\xi-t}{\varepsilon}, \eta\right)\left(\tilde{f}_{1}(t)+\tilde{f}_{2}(t)+\tilde{f}_{3}(t)\right) d t
\end{aligned}
$$

$$
\sqrt{2 \pi} \widetilde{R}_{\eta}(\xi, \eta)=\frac{1}{\varepsilon} \int_{-\infty}^{\infty} G_{\eta}\left(\frac{\xi-t}{\varepsilon}, \eta\right) \tilde{f}(t) d t
$$

We set $X=(\widetilde{U}, \widetilde{R})$ and let the left-hand side of $(4.19 \mathrm{a})-(4.19 \mathrm{~b})$ be denoted by $\mathscr{M}_{1}(X)(\xi)$ and $\mathscr{M}_{2}(X)(\xi)$ and the right-hand side by $\mathscr{N}_{1}(X ; \varepsilon)(\xi)$ and $\mathscr{N}_{2}(X ; \varepsilon)(\xi)$, respectively. Let $\mathscr{M}(X)=\left(\mathscr{M}_{1}(X), \mathscr{M}_{2}(X)\right), \mathscr{N}(X ; \varepsilon)=$ $\left(\mathscr{N}_{1}(X ; \varepsilon), \mathscr{N}_{2}(X ; \varepsilon)\right)$ so that $(4.19 \mathrm{a})-(4.19 \mathrm{~b})$ may be written as $\mathscr{M}(X)=$ $\mathscr{N}(X ; \varepsilon)$. We consider $X$ in a small neighborhood $W$ of $X_{0}=\left(\widetilde{U}_{0}, 0\right)$ in $D \times F$, where $\widetilde{U}_{0}$ is given in (4.14). The maps $\mathscr{M}$ and $\mathscr{N}$ will have ranges in $E \times F$. Since $\mathscr{N}_{2}$ must be measured in the space $F$, we need expressions for $\partial \mathscr{N}_{2} / \partial \xi$ and $\partial \mathscr{N}_{2} / \partial \eta$ and these are given by $(4.19 \mathrm{c})-(4.19 \mathrm{~d})$, respectively.

In our previous arguments, we have assumed that $\varepsilon$ was small and positive, and we now extend $\mathscr{N}$ by setting

$$
\mathscr{N}(X ; \varepsilon)=\left(\widetilde{R}(\xi, 0)\left\{(1-\rho)-3 \tilde{U}(\xi)-\frac{3}{2} \widetilde{R}(\xi, 0)\right\}, 0\right)
$$

for $\varepsilon \leq 0$. Our main task now is to show that $\mathcal{N}$ is continuous as $\varepsilon \rightarrow 0+$, and this we do in a sequence of calculations. By $(4.10 \mathrm{c})$

$$
\begin{aligned}
\tilde{f}_{3}(\xi)= & \varepsilon^{-1} f_{3}(\xi / \varepsilon) \\
= & (2 \varepsilon)^{-1}\left[\left\{\left(1+\varepsilon \widetilde{U}(\xi)+\varepsilon \widetilde{R}_{\eta}(\xi, 0)\right)^{2}+\varepsilon^{4}\left(\widetilde{U}^{\prime}(\xi)+\widetilde{R}_{\xi}(\xi, 0)\right)^{2}\right\}^{-1}\right. \\
& \left.\quad-1+2 \varepsilon \widetilde{U}(\xi)+2 \varepsilon \widetilde{R}_{\eta}(\xi, 0)\right] .
\end{aligned}
$$

From this explicit formula the following estimates are immediate for $(\widetilde{U}, \widetilde{R}) \in$ $W$ and $\varepsilon>0$ sufficiently small, say $\varepsilon \in\left(0, \varepsilon_{0}\right]$ :

$$
\left|\tilde{f}_{3}\right|_{E}+\varepsilon^{-1}\left|\tilde{f}_{3}-\frac{3}{2} \varepsilon(\widetilde{U}+\widetilde{R}(\cdot, 0))^{2}\right|_{E} \leq \text { const. } \varepsilon \text {. }
$$


Hence, the quantities estimated tend to zero as $\varepsilon \rightarrow 0+$, and we may extend them continuously to $\varepsilon \leq 0$. It is also clear that the Fréchet derivative with respect to $(\widetilde{U}, \widetilde{R})$ is continuous.

The next term we consider is $\tilde{f}_{2}$ defined by $\tilde{f}_{2}(\xi)=\varepsilon^{-1} f_{2}(\xi / \varepsilon)$ where $f_{2}$ is given in (4.10b). In that expression let us begin with the $\nabla \psi_{+}$term; it contributes

$$
\begin{aligned}
& \left|\nabla \psi_{+}\left(Q_{1}\left(\frac{\xi}{\varepsilon}, 0\right), Q_{2}\left(\frac{\xi}{\varepsilon}, 0\right)\right)\right|^{2}=\left|\nabla \psi_{+}\left(\frac{\xi}{\varepsilon}+S_{1}\left(\frac{\xi}{\varepsilon}, 0\right), S_{2}\left(\frac{\xi}{\varepsilon}, 0\right)\right)\right|^{2} \\
& \quad=\left|\nabla \psi_{+}\left(\frac{1}{\varepsilon}\left(\xi+\varepsilon S_{1}\left(\frac{\xi}{\varepsilon}, 0\right)\right), \varepsilon(\widetilde{U}(\xi)+\widetilde{R}(\xi, 0))\right)\right|^{2} .
\end{aligned}
$$

If the remaining terms defining $f_{2}(\xi / \varepsilon)$ are subtracted, and a factor $\varepsilon^{-1}$ multiplied, then (3.20)-(3.21) may be used to yield

$$
\left|\tilde{f}_{2}\right|_{E} \leq\left|\tilde{f}_{2}\right|_{D} \leq \text { const. } \varepsilon^{3}
$$

for $(\widetilde{U}, \widetilde{R})$ in the neighborhood $W$ of $\left(\widetilde{U}_{0}, 0\right)$ in $D \times F$ and $\varepsilon \in\left(0, \varepsilon_{0}\right]$. A similar result holds for the Fréchet derivative.

The term involving $\tilde{f}_{1}$ is more complicated. Now $\tilde{f}_{1}(\xi)=\varepsilon^{-1} f_{1}(\xi / \varepsilon)$, where $f_{1}$ is given in (4.10a). The use of the formulae

$$
\begin{gathered}
\left(S_{2}\right)_{\xi}(t, 0)=\varepsilon^{2}\left(\widetilde{S}_{2}\right)_{\xi}(\varepsilon t, 0) \\
S_{1}(t, 0)-S_{1}(\xi, 0)=\int_{\xi}^{t}\left(S_{2}\right)_{\eta}(v, 0) d v=\varepsilon \int_{\xi}^{t}\left(\widetilde{S}_{2}\right)_{\eta}(\varepsilon v, 0) d v
\end{gathered}
$$

yields

(4.22)

$$
\varepsilon^{-1} \tilde{f}_{1}(\xi)=\frac{\rho}{\pi} \int_{-\infty}^{\infty}\left(\widetilde{S}_{2}\right)_{\xi}(t, 0)\left\{\left[t-\xi+\varepsilon \int_{\xi}^{t}\left(\widetilde{S}_{2}\right)_{\eta}(v, 0) d v\right]^{-1}-(t-\xi)^{-1}\right\} d t .
$$

In the Appendix we prove the important estimate

$$
\left|\tilde{f}_{1}\right|_{E} \leq \text { const. } \varepsilon^{2}
$$

for $(\widetilde{U}, \widetilde{R}) \in W$ and $\varepsilon>0$ sufficiently small. A similar result holds for the Fréchet derivative.

The use of (4.20), (4.21), and (4.23) together with Lemma 6 yields (4.24)

$$
\left|\mathscr{N}_{2}(X ; \varepsilon)\right|_{F}+\left|\mathscr{N}_{1}(X ; \varepsilon)-\widetilde{R}(\cdot, 0)\left(1-\rho-3 \widetilde{U}-\frac{3}{2} \widetilde{R}(\cdot, 0)\right)\right|_{E} \leq \text { const. } \varepsilon
$$

for $X=(\widetilde{U}, \widetilde{R}) \in W$ and $\varepsilon \in\left(0, \varepsilon_{0}\right]$. We can then extend $\mathscr{N}_{1}$ and $\mathscr{N}_{2}$ smoothly for $\varepsilon \in\left(-\varepsilon_{0}, 0\right)$ by setting them equal to their values at $\varepsilon=0$. The Fréchet derivatives $\mathscr{M}_{X}$ and $\mathscr{N}_{X}$ are also continuous for $(X, \varepsilon) \in W \times$ $\left(-\varepsilon_{0}, \varepsilon_{0}\right)$. Since we know that $\left(X_{0}, 0\right)$ satisfies $\mathscr{M}(X)-\mathscr{N}(X, 0)=0$ we shall have the existence of a branch for $\varepsilon \in\left(0, \varepsilon_{0}\right)$ as soon as we have shown that the linear operator $Y=\mathscr{M}_{X}\left(X_{0}\right)-\mathscr{N}_{X}\left(X_{0}, 0\right)$ is injective and surjective as a map from $D \times F$ to $E \times F$. 


\section{THE MAP $Y$ IS A HOMEOMORPHISM}

The linear map $Y$ evaluated at an arbitrary $(\widetilde{U}, \widetilde{R}) \in D \times F$ is given by

$$
\begin{aligned}
& \left(\rho H\left(\tilde{U}^{\prime}\right)(\xi)-(1-\rho) \widetilde{U}(\xi)+3 \widetilde{U}_{0}(\xi) \widetilde{U}(\xi)\right. \\
& \left.\quad-(1-\rho) \widetilde{R}(\xi, 0)+3 \widetilde{U}_{0}(\xi) \widetilde{R}(\xi, 0), \widetilde{R}(\xi, \eta)\right) .
\end{aligned}
$$

The special form of the second component ensures that we need only prove that the map $\widetilde{U} \mapsto \rho H\left(\widetilde{U}^{\prime}\right)-(1-\rho) \widetilde{U}+3 \widetilde{U}_{0} \widetilde{U}$ is a homeomorphism from $D$ to $E$. After scaling the dependent variable, we may work with the operator

$$
L u(x)=H\left(u^{\prime}\right)(x)-u(x)+\frac{4 u(x)}{1+x^{2}} .
$$

If $L$ were not injective, then unity would be an eigenvalue of the map $H\left(u^{\prime}\right)(x)$ $+4 u(x) /\left(1+x^{2}\right)$ considered as an unbounded, selfadjoint map on $W^{1,2}(\mathbb{R})$. However, it is known that $(0, \infty)$ is the continuous spectrum [11, p. 358]; hence $L$ is injective.

We now show that $L$ is surjective; let $g \in E$ and consider the equation

$$
-H\left(u^{\prime}\right)(x)+u(x)=\frac{4 u(x)}{1+x^{2}}-g(x) .
$$

The operator on the left may be inverted [6], and there results

$$
u(x)=(\mathscr{C} u)(x)+l(g)(x)
$$

where

$$
\begin{gathered}
(\mathscr{C} u)(x)=4 \int_{-\infty}^{\infty} K(x-t) \frac{u(t)}{1+t^{2}} d t \\
l(g)(x)=-\int_{-\infty}^{\infty} K(x-t) g(t) d t
\end{gathered}
$$

The kernel $K$ is given by

$$
K(t)=\frac{1}{\pi} \int_{0}^{\infty} \frac{s e^{-s}}{t^{2}+s^{2}} d s
$$

note that

$$
K(t) \sim-\frac{1}{\pi} \log |t| \quad \text { as } t \rightarrow 0
$$

and

$$
K(t)=\frac{1}{\pi t^{2}} \int_{0}^{\infty} s e^{-s} d s+O\left(t^{-4}\right)=\frac{1}{\pi t^{2}}+O\left(t^{-4}\right) \quad \text { as } t \rightarrow \infty .
$$

One obtains similar expressions for $K^{\prime}$; in particular, $K^{\prime}(t) \sim-(\pi t)^{-1}$ as $t \rightarrow 0$ and $K^{\prime}(t) \sim-2\left(\pi t^{3}\right)^{-1}$ as $t \rightarrow \infty$. The use of this together with Privalov's theorem [12] ensures that $\mathscr{C}$ and $l$ are bounded, linear maps from $E$ to $D$, and, therefore, a fortiori, from $E$ to $E$. Given $g \in E$, it suffices to solve (5.2) for $u \in E$, since then $\mathscr{C} u \in D$, whence $u \in D$. We know that unity is not an eigenvalue of $\mathscr{C}: E \rightarrow E$ since this is equivalent to $L$ being injective. In the next lemma, we show that $\mathscr{C}$ is a compact, linear map so that $I-\mathscr{C}$ is surjective by Riesz-Schauder theory. The compactness of $\mathscr{C}$ is due to the weight $\left(1+t^{2}\right)^{-1}$ in the integrand. 
Lemma 7. The linear map $\mathscr{C}: E \rightarrow E$ is compact; that is, if $\left\{u_{n}\right\}_{n=1}^{\infty}$ is a sequence with $\left|u_{n}\right|_{E} \leq 1$, then $\left\{\mathscr{C} u_{n}\right\}$ has a convergent subsequence in $E$.

Proof. As noted earlier $\left\{\mathscr{C} u_{n}\right\}$ is bounded in the space $D$, which is just $C^{1+\alpha}(\mathbb{R})$ with the weight $1+x^{2}$. The imbedding of $C^{1+\alpha}(I)$ into $C^{\alpha}(I)$ is compact for any bounded, open interval $I$, whence we may assume, after choosing a suitable subsequence if necessary, that $\mathscr{C} u_{n}$ converges in $C^{\alpha}(I)$ to some $v \in E$. To complete the proof, we must show the convergence of $\mathscr{C} u_{n}$ to $v$ in $E$, that is with the weight:

$$
\sup _{x \in \mathbb{R}}\left(1+x^{2}\right)\left[\mathscr{C} u_{n}-v\right](x) \rightarrow 0 \text { as } n \rightarrow \infty .
$$

We begin by showing that

$$
\lim _{|x| \rightarrow \infty}\left(1+x^{2}\right)\left|\int_{-\infty}^{\infty} K(x-t) \frac{u_{n}(t)}{1+t^{2}} d t-\frac{1}{\pi x^{2}} \int_{-\infty}^{\infty} \frac{u_{n}(t)}{1+t^{2}} d t\right|=0 .
$$

We shall consider the case $x \rightarrow \infty$ since that for $x \rightarrow-\infty$ is similar. Now

$$
\begin{aligned}
\int_{x+1 / 2}^{\infty} K(x-t) \frac{\left|u_{n}(t)\right|}{1+t^{2}} d t \leq \text { const. } \int_{x+1 / 2}^{\infty} \frac{d t}{(x-t)^{2}\left(1+t^{4}\right)} \leq \frac{\text { const. }}{x^{4}} \\
\int_{x-1 / 2}^{x+1 / 2} K(x-t) \frac{\left|u_{n}(t)\right|}{1+t^{2}} d t \leq \text { const. } \int_{x-1 / 2}^{x+1 / 2} \log \frac{1}{|x-t|} \frac{d t}{1+t^{4}} \\
\leq \frac{\text { const. }}{x^{4}}
\end{aligned}
$$

and

$$
\int_{x-1 / 2}^{\infty} \frac{u_{n}(t)}{1+t^{2}} d t \leq \frac{\text { const. }}{x^{3}}
$$

The use of (5.3) yields

$$
\begin{aligned}
& \mid \int_{-\infty}^{x-1 / 2} K(x-t) \frac{u_{n}(t)}{1+t^{2}} d t-\frac{1}{\pi x^{2}} \int_{-\infty}^{x-1 / 2} \frac{u_{n}(t)}{1+t^{2}} d t \\
& \quad-\frac{1}{\pi} \int_{-\infty}^{x-1 / 2}\left\{\frac{1}{(x-t)^{2}}-\frac{1}{x^{2}}\right\} \frac{u_{n}(t)}{1+t^{2}} d t \mid \\
& \leq \text { const. } \int_{-\infty}^{x-1 / 2} \frac{1}{(x-t)^{4}} \frac{d t}{1+t^{4}} \leq \text { const. } x^{-4}
\end{aligned}
$$

Finally,

$$
\begin{aligned}
\int_{-\infty}^{x-1 / 2}\left|\frac{1}{(x-t)^{2}}-\frac{1}{x^{2}}\right| \frac{\left|u_{n}(t)\right|}{1+t^{2}} d t & \leq \text { const. } \frac{1}{x^{2}} \int_{-\infty}^{x-1 / 2} \frac{\left|2 \frac{t}{x}-\frac{t^{2}}{x^{2}}\right|}{\left(1-\frac{t}{x}\right)^{2}} \frac{d t}{1+t^{4}} \\
& \leq \text { const. } x^{-1},
\end{aligned}
$$

and the use of this with (5.5)-(5.6) yields (5.4).

To control the Hölder estimate, we now prove that

$$
\varlimsup_{|x| \rightarrow \infty}\left(1+|x|^{3}\right)\left|\int_{-\infty}^{\infty} K_{x}(x-t) \frac{u_{n}(t)}{1+t^{2}} d t\right| \leq \text { const. }
$$


and shall do the case $x \rightarrow \infty$. Now

$$
\begin{aligned}
\int_{x+1 / 2}^{\infty}\left|K_{x}(x-t)\right| \frac{\left|u_{n}(t)\right|}{1+t^{2}} d t & \leq \text { const. } \int_{x+1 / 2}^{\infty} \frac{d t}{|x-t|^{3}\left(1+t^{4}\right)} \\
& \leq \text { const. } x^{-4} .
\end{aligned}
$$

On the interval $\left(x-\frac{1}{2}, x+\frac{1}{2}\right), K_{x}(x-t) \sim-\pi^{-1}(x-t)^{-1}$ so

$$
\begin{aligned}
\left|\int_{x-1 / 2}^{x+1 / 2} K_{x}(x-t) \frac{u_{n}(t)}{1+t^{2}} d t\right| & \sim \frac{1}{\pi}\left|\int_{x-1 / 2}^{x+1 / 2} \frac{1}{x-t}\left\{\frac{u_{n}(t)}{1+t^{2}}-\frac{u_{n}(x)}{1+x^{2}}\right\} d t\right| \\
& \leq \text { const. } x^{-4}
\end{aligned}
$$

where the integral is a principal value. The last term is estimated by

$$
\begin{aligned}
\left|\int_{-\infty}^{x-1 / 2} K_{x}(x-t) \frac{u_{n}(t)}{1+t^{2}} d t\right| & \leq \text { const. } \int_{-\infty}^{x-1 / 2} \frac{d t}{|x-t|^{3}\left(1+t^{4}\right)} \\
& \leq \text { const. } x^{-3}
\end{aligned}
$$

which together with (5.8) and (5.9) yields (5.7). It follows from (5.7) that

$$
\left|\frac{d}{d x} \mathscr{C} u_{n}(x)\right| \leq \frac{\text { const. }}{1+|x|^{3}}, \quad x \in \mathbb{R},
$$

which easily gives

$$
\lim _{|x| \rightarrow \infty}\left(1+x^{2}\right) \sup _{\substack{\tilde{x} \in \mathbb{R} \\|x-\tilde{x}| \leq 1}} \frac{\left|\mathscr{C} u_{n}(x)-\mathscr{C} u_{n}(\tilde{x})\right|}{|x-\tilde{x}|^{\alpha}}=0 .
$$

Since $\left|u_{n}\right|_{E} \leq 1$, we have $\left|u_{n}(t)\right| \leq\left(1+t^{2}\right)^{-1}$, and so we may assume without loss of generality that the sequence of numbers

$$
\int_{-\infty}^{\infty} \frac{u_{n}(t)}{1+t^{2}} d t
$$

has a limit as $n \rightarrow \infty$. The use of this with (5.4) and (5.10) together with the convergence of $\left\{\mathscr{C} u_{n}\right\}$ in $C^{\alpha}(I), I$ bounded, ensures that $\left\{\mathscr{C} u_{n}\right\}$ is a Cauchy sequence in the Banach space $E$.

This lemma together with our earlier results proves that $L$ is a homeomorphism, whence $\mathscr{M}_{X}\left(X_{0}\right)-\mathscr{N}_{X}\left(X_{0} ; \varepsilon\right)$ is a homeomorphism.

Theorem 8. There exists $\varepsilon_{0}>0$ such that for each $\varepsilon \in\left(0, \varepsilon_{0}\right)$ the equations (4.19a)-(4.19b) have a unique solution (modulo translation) $(\widetilde{U}, \widetilde{R}) \in D \times F$ with $\widetilde{U}(\xi)=\widetilde{U}(\xi ; \varepsilon), \widetilde{R}(\xi, \eta)=\widetilde{R}(\xi, \eta ; \varepsilon)$, continuous functions of $\varepsilon$. Furthermore,

$$
\left|\widetilde{U}(\cdot ; \varepsilon)-\widetilde{U}_{0}(\cdot)\right|_{D}+|\widetilde{R}(\cdot, \cdot ; \varepsilon)|_{F} \leq \text { const. } \varepsilon .
$$

Proof. The first part follows from the implicit function theorem, while (5.11) follows from the estimates for $\tilde{f}_{1}, \tilde{f}_{2}, \tilde{f}_{3}$, and $\tilde{f}_{4}$ used in (4.19).

Remarks. (i) We could have sought solutions $(\widetilde{U}, \widetilde{R})$ to $(4.19 \mathrm{a})-(4.19 \mathrm{~b})$ which were even functions of $\xi$, and our preceding estimates would have provided them. Since we have a uniqueness result in Theorem 8 , we may assume, upon 
choosing $\varepsilon_{0}$ smaller if necessary, that $(\widetilde{U}, \widetilde{R})$ are even functions after a suitable translation.

(ii) The functions $\widetilde{U}(\cdot)$ and $\widetilde{R}(\cdot, 0)$ are in $C^{1+\alpha}(\mathbb{R})$, and we may iterate our estimates to show that these functions, and therefore the function $Z$ defining the interface $\Gamma$, are of class $C^{\infty}$. Standard arguments [8] ensure that $Z$ is real-analytic.

(iii) As noted in (4.1), the interface $\Gamma$ is given by

$$
\left\{\left(Q_{1}(x, 0), Q_{2}(x, 0)\right): x \in \mathbb{R}\right\}
$$

where

$$
\begin{aligned}
Q_{1}(x, 0) & =x+S_{1}(x, 0)=x+\int_{0}^{x}\left(S_{2}\right)_{\eta}(s, 0) d s \\
& =x+\varepsilon \int_{0}^{x}\left\{\widetilde{U}(\varepsilon s ; \varepsilon)+\widetilde{R}_{\eta}(\varepsilon s, 0 ; \varepsilon)\right\} d s
\end{aligned}
$$

and

$$
\begin{aligned}
Q_{2}(x, 0) & =\varepsilon \widetilde{U}(\varepsilon x ; \varepsilon)+\varepsilon \widetilde{R}(\varepsilon x ; \varepsilon) \\
& =\varepsilon \widetilde{U}_{0}(\varepsilon x)+\varepsilon\left(\widetilde{U}(\varepsilon x ; \varepsilon)-\widetilde{U}_{0}(\varepsilon x)+\widetilde{R}(\varepsilon x ; \varepsilon)\right)
\end{aligned}
$$

If we set $t=Q_{1}(x, 0)$ and $Z(t)=Q_{2}(x, 0)$, and use (5.11)-(5.12), there results

$$
Z(t)=\varepsilon \widetilde{U}_{0}(\varepsilon t)+O\left(\frac{\varepsilon^{2}}{1+\varepsilon^{2} t^{2}}\right)
$$

where $\tilde{U}_{0}$ is given by (4.14) and $\varepsilon>0$ is related to the speed $c$ by $\varepsilon=$ $g c_{0}^{-2}-g c^{-2}$ with $c_{0}=\sqrt{(1-\rho) g}$.

\section{APPENDIX}

Proof of Lemma 3. Let $h=\left(h_{1}, h_{2}\right) \in B \times B$, and write $(x, y)$ for $\left(x_{1}, x_{2}\right)$. It is clear from (3.13) that

$$
\begin{aligned}
|\Gamma(h)(\tilde{x}, \tilde{y})| \leq \text { const. } \tilde{y}\|h\| \int_{T_{+}}\left(1+r^{4}\right)^{-1}\left\{(x-\tilde{x})^{2}+(y-\tilde{y})^{2}\right\}^{-1 / 2} \\
\times\left\{(x-\tilde{x})^{2}+(y+\tilde{y})^{2}\right\}^{-1 / 2} d x d y
\end{aligned}
$$

where $r^{2}=x^{2}+y^{2}$ and $\|h\|=\left|h_{1}\right|_{B}+\left|h_{2}\right|_{B}$. Let $\tilde{x}=\rho \cos \phi, \tilde{y}=\rho \sin \phi$, $\phi \in(0, \pi)$, and use the Schwarz inequality to obtain

$$
\begin{aligned}
|\Gamma(h)(\tilde{x}, \tilde{y})| \leq & \text { const. } \tilde{y}\|h\| \int_{0}^{\infty} \frac{r d r}{1+r^{4}}\left\{\int_{0}^{\pi} \frac{d \theta}{r^{2}+\rho^{2}-2 \rho r \cos (\theta-\phi)}\right\}^{1 / 2} \\
& \times\left\{\int_{0}^{\pi} \frac{d \theta}{r^{2}+\rho^{2}-2 \rho r \cos (\theta+\phi)}\right\}^{1 / 2} .
\end{aligned}
$$


The first $\theta$-integral may be evaluated via Poisson's integral formula:

$$
\begin{aligned}
|\Gamma(h)(\tilde{x}, \tilde{y})| \leq & \text { const. } \tilde{y}\|h\| \int_{0}^{\infty} \frac{r d r}{\left(1+r^{4}\right) \sqrt{\left|r^{2}-\rho^{2}\right|}} \\
& \times\left\{\int_{0}^{\pi} \frac{d \theta}{r^{2}+\rho^{2}-2 \rho r \cos (\theta+\phi)}\right\}^{1 / 2} \\
= & \text { const. } \tilde{y}\|h\| \int_{0}^{\infty} \frac{t d t}{\left(1+\rho^{4} t^{4}\right) \sqrt{\left|1-t^{2}\right|}} \\
& \times\left\{\int_{0}^{\pi} \frac{d \theta}{t^{2}+1-2 t \cos (\theta+\phi)}\right\}^{1 / 2} .
\end{aligned}
$$

We write the integral with respect to $t$ as a sum over the intervals $(0,1 / 3)$, $(3, \infty)$, and $(1 / 3,3)$. The first and second are dominated easily by

$$
\frac{\text { const. } \tilde{y}\|h\|}{1+\rho^{2}} \text { and } \frac{\text { const. } \tilde{y}\|h\|}{1+\rho^{4}}
$$

respectively. The inequality

$$
\begin{aligned}
\int_{0}^{\pi} \frac{d \theta}{t^{2}+1-2 t \cos (\theta+\phi)} & =\int_{0}^{\pi} \frac{d \theta}{(t-1)^{2}+2 t(1-\cos (\theta+\phi))} \\
& \leq \frac{3}{2} \int_{0}^{\pi} \frac{d \theta}{1-\cos (\theta+\phi)} \leq \text { const. }\left(\frac{1}{\phi}+\frac{1}{\pi-\phi}\right)
\end{aligned}
$$

for $t \in(1 / 3,3)$ allows us to bound the third term by

$$
\frac{\text { const. } \tilde{y}\|h\|}{1+\rho^{4}}\left(\frac{1}{\phi}+\frac{1}{\pi-\phi}\right)^{1 / 2} \leq \frac{\text { const. } \sqrt{y}\|h\|}{1+\rho^{7 / 2}} \leq \frac{\text { const. }(1+\tilde{y})\|h\|}{1+\rho^{2}} .
$$

The use of this with (A.1) yields

$$
|\Gamma(h)(\tilde{x}, \tilde{y})| \leq \frac{\text { const. }(1+\tilde{y})\|h\|}{1+\tilde{x}^{2}+\tilde{y}^{2}}
$$

for all $(\tilde{x}, \tilde{y}) \in T_{+}$.

Since $\Delta \Gamma(h)=\operatorname{div} h$ and $\Gamma(x, 0)=0$, standard elliptic theory [13] gives

$|\Gamma(h)|_{C^{1+\alpha}([x-1, x+1] \times[0,1])}$

$$
\leq \text { const. }\left\{|\Gamma(h)|_{L_{\infty}([x-2, x+2] \times[0,2])}+|h|_{C^{\alpha}([x-2, x+2] \times[0,2])}\right\} \leq \frac{\text { const. }}{1+x^{2}} .
$$

Interior estimates are similar to those in Lemma 1.

Proof of Lemma 5. Since $\widehat{G}(k, y)$ given by (4.16) is even in $k$, we give most estimates for $k \geq 0$. The following estimates hold:

(A.3a) $\widehat{G}(k, \eta)=\frac{-(\eta+1)}{3 \rho^{2}}+(\eta+1) O(k), \quad k \in[0,1], \eta \in[-1,0]$,

(A.3b) $\widehat{G}(k, \eta)=\frac{-(\eta+1)}{\rho k}+(\eta+1) O\left(e^{k \eta}\right), \quad k \in(1, \infty), \eta \in[-1,-1 / 2]$,

$$
\begin{aligned}
\widehat{G}(k, \eta)=\frac{e^{k \eta}}{k(1+\rho)}\left(1+\frac{1}{k(1+\rho)}\right)-\frac{(\eta+1)}{\rho k}+O\left(k^{-3} e^{k \eta}\right), & \\
k & \in(1, \infty), \eta \in[-1 / 2,0] .
\end{aligned}
$$


Similar estimates hold for derivatives of $\widehat{G}$ with respect to $k$ and $\eta$. Now

$$
G(x, \eta)=\sqrt{\frac{2}{\pi}} \int_{0}^{\infty} \widehat{G}(k, \eta) \cos k x d k
$$

and two integrations by parts easily give part (a) of the lemma.

To prove (b), we restrict attention to $|x| \leq 1 / 2$. If $\eta \in[-1,-1 / 2]$, then (A.3a)-(A.3b) yield

$$
\begin{aligned}
G(x, \eta) & =(1+\eta)\left\{O(1)-\sqrt{\frac{2}{\pi}} \int_{1}^{\infty} \frac{\cos k x}{\rho k} d k\right\} \\
& =(1+\eta)\left\{O(1)+\frac{1}{\rho} \sqrt{\frac{2}{\pi}} \log |x|\right\}
\end{aligned}
$$

with similar estimates for $G_{x}$ and $G_{\eta}$. If $\eta \in\left[-\frac{1}{2}, 0\right)$ then (A.3a), (A.3c) and a bit of calculus yield

$$
\begin{aligned}
G(x, \eta)= & O(1)+\sqrt{\frac{2}{\pi}} \int_{1}^{\infty} \cos k x \frac{e^{k \eta}}{k(1+\rho)}\left\{1+\frac{1}{k(1+\rho)}+O\left(k^{-2}\right)\right\} d k \\
& -\sqrt{\frac{2}{\pi}} \frac{(\eta+1)}{\rho} \int_{|x|}^{\infty} \frac{\cos t}{t} d t \\
= & O(1)+\sqrt{\frac{2}{\pi}} \frac{1}{1+\rho} \int_{-\eta}^{-\eta /|x|} \frac{e^{-s}}{s} d s+\frac{(1+\eta)}{\rho} \sqrt{\frac{2}{\pi}} \log |x| \\
= & O(1)+\sqrt{\frac{2}{\pi}} \frac{1}{1+\rho}\left\{e^{\eta /|x|} \log \left|\frac{\eta}{x}\right|-e^{\eta} \log |\eta|\right\}+\frac{(1+\eta)}{\rho} \sqrt{\frac{2}{\pi}} \log |x| \\
= & O(1)-\sqrt{\frac{2}{\pi}} \frac{(1+\eta)}{1+\rho} \log \sqrt{x^{2}+\eta^{2}}+\frac{(1+\eta)}{\rho} \sqrt{\frac{2}{\pi}} \log |x| .
\end{aligned}
$$

Since $\eta<0$, we can differentiate with respect to $x$ or $\eta$ within the integral over $(1, \infty)$. There results

$$
\begin{aligned}
G_{x}(x, \eta)= & O(1)-\sqrt{\frac{2}{\pi}} \int_{1}^{\infty} \sin k x \frac{e^{k \eta}}{1+\rho}\left\{1+\frac{1}{k(1+\rho)}+O\left(k^{-2}\right)\right\} d k \\
& +\sqrt{\frac{2}{\pi}} \frac{(1+\eta)}{\rho x} \\
= & O(1)+\sqrt{\frac{2}{\pi}}(1+\eta)\left\{\frac{1}{\rho x}-\frac{x}{(1+\rho)\left(x^{2}+\eta^{2}\right)}\right\} ; \\
G_{\eta}(x, \eta)= & O(1)+\sqrt{\frac{2}{\pi}} \int_{1}^{\infty} \cos k x \frac{e^{k \eta}}{1+\rho}\left\{1+\frac{1}{k(1+\rho)}+O\left(k^{-2}\right)\right\} d k \\
& +\frac{1}{\rho} \sqrt{\frac{2}{\pi}} \log |x| \\
= & O(1)-\sqrt{\frac{2}{\pi}} \frac{1}{1+\rho}\left\{\frac{\eta}{\eta^{2}+x^{2}}+\log \sqrt{x^{2}+\eta^{2}}\right\}+\sqrt{\frac{2}{\pi}} \frac{1}{\rho} \log |x| .
\end{aligned}
$$

The proof of (c) is immediate from Lemma 6 with $\varepsilon=1$. 
Proof of Lemma 6. We first prove the result for $\left[\mathscr{L}_{\varepsilon} g\right]$. The use of Lemma 5 yields

$$
\begin{aligned}
& \left|\mathscr{L}_{\varepsilon} g(x, \eta)\right| \\
& \quad \leq \frac{\text { const. }}{\varepsilon}|g|_{E}\left\{\int_{x-\varepsilon / 2}^{x+\varepsilon / 2}-\log \left|\frac{x-t}{\varepsilon}\right| \frac{d t}{1+t^{2}}+\int_{|x-t| \geq \varepsilon / 2} \frac{\varepsilon^{2}}{(x-t)^{2}} \frac{d t}{1+t^{2}}\right\} \\
& \quad \leq \frac{\text { const. }}{1+x^{2}}|g|_{E} .
\end{aligned}
$$

For the Hölder term in $\left[\mathscr{L}_{\varepsilon} g\right]$, it suffices to do a variation in the $x$-direction because of the results for $\left[\partial \mathscr{L}_{\varepsilon} g / \partial \eta\right]$. Now

$$
\begin{aligned}
& \mid \mathscr{L}_{\varepsilon} g(\tilde{x}, \eta)-\mathscr{L}_{\varepsilon} g(x, \eta) \mid \leq \text { const. }\left\{\int_{|t| \geq 1 / 2}|g(\varepsilon t+\tilde{x})-g(\varepsilon t+x)| \frac{d t}{t^{2}}\right. \\
&\left.\qquad-\int_{-1 / 2}^{1 / 2} \log |t||g(\varepsilon t+\tilde{x})-g(\varepsilon t+x)| d t\right\} \\
& \leq \text { const. }|g|_{E}|x-\tilde{x}|^{\alpha}\left\{\int_{|t| \geq 1 / 2} \frac{1}{t^{2}} \frac{1}{1+(\varepsilon t+x)^{2}} d t+\frac{1}{1+x^{2}}\right\} \\
& \leq \frac{\text { const. }}{1+x^{2}}|g|_{E}|x-\tilde{x}|^{\alpha} .
\end{aligned}
$$

Next we estimate

$$
\varepsilon \frac{\partial}{\partial x} \mathscr{L}_{\varepsilon} g(x, \eta)=\frac{1}{\varepsilon} \int_{-\infty}^{\infty} G_{x}\left(\frac{x-t}{\varepsilon}, \eta\right) g(t) d t,
$$

and begin by noting that the integral over $(-\infty, x-\varepsilon / 2) \cup(x+\varepsilon / 2, \infty)$ is estimated with the aid of Lemma $5(a)$ as before. Now

$$
\begin{aligned}
& \frac{1}{\varepsilon} \int_{x-\varepsilon / 2}^{x+\varepsilon / 2} G_{x}\left(\frac{x-t}{\varepsilon}, \eta\right) g(t) d t \\
& \quad=\sqrt{\frac{2}{\pi}}(1+\eta) \int_{x-\varepsilon / 2}^{x+\varepsilon / 2}\left\{\frac{\rho}{x-t}-\frac{1}{1+\rho} \frac{x-t}{(x-t)^{2}+\eta^{2} \varepsilon^{2}}+O(1)\right\}(g(t)-g(x)) d t,
\end{aligned}
$$

and the use of this with the estimate $|g(t)-g(x)| \leq|g|_{E}|t-x|^{\alpha} /\left(1+x^{2}\right)$ ensures that the term in (A.4) has amplitude at most

$$
\frac{\text { const. }}{1+x^{2}}|g|_{E} \varepsilon^{\alpha} \text {. }
$$

For the Hölder estimate, one needs to estimate integrals only over $(x-\varepsilon / 2, x+$ $\varepsilon / 2$ ), and the result is immediate from Lemma $5(b)$ together with the proof of Privalov's theorem [12].

For the $\partial \mathscr{L}_{\varepsilon} g / \partial \eta$ term, the important part of the expression is

$$
\int_{-1 / 2}^{1 / 2} \frac{\eta}{t^{2}+\eta^{2}} g(\varepsilon t+x) d t
$$

which has magnitude bounded by 


$$
\frac{\text { const. }}{1+x^{2}}|g|_{E} \text {. }
$$

Variations of (A.5) with respect to $x$ or $\eta$ immediately give the desired result for the Hölder estimate.

Proof of (4.23). For $u, w \in E$ and small $\varepsilon$ define

$$
F(x)=\int_{-\infty}^{\infty} \frac{w(s) \int_{x}^{s} u(t) d t}{\left(s-x+\varepsilon \int_{x}^{s} u(t) d t\right)(s-x)} d s, \quad x \in \mathbb{R},
$$

where the integral is as a principal value. In order to prove (4.23), it suffices, due to (4.22), to show that

$$
|F|_{E} \leq \text { const. }|u|_{E}|w|_{E}
$$

for $|u|_{E} \leq 1$. The integral over $(-\infty, x-1) \cup(x+1, \infty)$ may be bounded by

$$
\text { const. }|u|_{E}|w|_{E} \int_{|s-x| \geq 1} \frac{d s}{(s-x)^{2}\left(1+s^{2}\right)} \leq \frac{\text { const. }}{1+x^{2}}|u|_{E}|w|_{E}
$$

The integral over $(x-1, x+1)$ may be written as

$$
\begin{gathered}
\int_{x-1}^{x+1} \frac{w(s) \int_{x}^{s}(u(t)-u(x)) d t}{\left(s-x+\varepsilon \int_{x}^{s} u(t) d t\right)(s-x)} d s+u(x) \int_{x-1}^{x+1} \frac{w(s)-w(x)}{s-x+\varepsilon \int_{x}^{s} u(t) d t} d s \\
+u(x) w(x) \int_{-1}^{1}\left\{s+\varepsilon s u(x)+\varepsilon \int_{x}^{s+x}(u(t)-u(x)) d t\right\}^{-1} d s
\end{gathered}
$$

The first two terms may be bounded by

$$
\frac{\text { const. }}{1+x^{2}}|u|_{E}|w|_{E},
$$

while the integral over $(-1,1)$ equals

$$
\int_{-1}^{1}\left\{s+\varepsilon s+\varepsilon O\left(s^{1+\alpha}\right)\right\}^{-1} d s
$$

which is bounded as a principal value (indeed, it is $O(\varepsilon)$ ). The use of this with (A.7)-(A.9) yields

$$
|F(x)| \leq \frac{\text { const. }}{1+x^{2}}|u|_{E}|w|_{E}
$$

which is half of what we need to prove in (A.6).

We must estimate the Hölder norm of $F$. Now for $|x-y| \leq 1$,

$$
\begin{aligned}
F(x)-F(y)= & \int_{-\infty}^{\infty} \frac{w(s+x)}{s+\varepsilon \int_{x}^{s+x} u}\left(\frac{\int_{x}^{s+x} u}{s}\right) d s \\
& -\int_{-\infty}^{\infty} \frac{w(s+y)}{s+\varepsilon \int_{y}^{s+y} u}\left(\frac{\int_{y}^{s+y} u}{s}\right) d s
\end{aligned}
$$


which we rewrite as the sum of three terms:

$$
\begin{aligned}
\int_{-\infty}^{\infty} & \frac{w(s+x)-w(s+y)}{s+\varepsilon \int_{x}^{s+x} u}\left(\frac{\int_{x}^{s+x} u}{s}\right) d s \\
& +\int_{-\infty}^{\infty} \frac{w(s+y)}{s+\varepsilon \int_{x}^{s+x} u}\left(\frac{\int_{x}^{s+x} u-\int_{y}^{s+y} u}{s}\right) d s \\
& +\int_{-\infty}^{\infty} w(s+y)\left\{\frac{1}{s+\varepsilon \int_{x}^{s+x} u}-\frac{1}{s+\varepsilon \int_{y}^{s+y} u}\right\}\left(\frac{\int_{y}^{s+y}}{s}\right) d s .
\end{aligned}
$$

The first term may be rewritten as

$$
\begin{aligned}
\int_{-\infty}^{\infty} & \frac{w(s+x)-w(s+y)}{s+\varepsilon \int_{x}^{s+x} u}\left(\frac{\int_{x}^{s+x}(u(t)-u(x)) d t}{s}\right) d s \\
& +u(x) \int_{-\infty}^{\infty}\{w(s+x)-w(s+y)\}\left(\frac{1}{s+\varepsilon \int_{x}^{s+x} u}-\frac{1}{s+\varepsilon s u(x)}\right) d s \\
& +\frac{u(x)}{1+\varepsilon u(x)} \int_{-\infty}^{\infty} \frac{w(s+x)-w(s+y)}{s} d s .
\end{aligned}
$$

The first two terms are easily bounded by

$$
\frac{\text { const. }}{1+x^{2}}|u|_{E}|w|_{E}|x-y|^{\alpha}
$$

and the same holds for the last by Privalov's theorem [12].

We estimate the second integral in (A.11); it is given by

$$
\begin{gathered}
\int_{-\infty}^{\infty} w(s+y)\left\{\frac{1}{s+\varepsilon \int_{x}^{s+x} u}-\frac{1}{s+\varepsilon u(x) s}\right\}\left\{\frac{\int_{0}^{s}(u(x+t)-u(y+t)) d t}{s}\right\} d s \\
+\frac{1}{1+\varepsilon u(x)} \int_{-\infty}^{\infty} \frac{w(s+y)-w(y)}{s}\left\{\frac{\int_{0}^{s}(u(x+t)-u(y+t)) d t}{s}\right\} d s \\
+\frac{w(y)}{1+\varepsilon u(x)} \int_{0}^{\infty} s^{-2} \int_{0}^{s}(u(x+t)-u(y+t)-u(x-t)+u(y-t)) d t d s
\end{gathered}
$$

Since

(A.13a)

$$
\begin{aligned}
& \left|\int_{0}^{s}(u(x+t)-u(y+t)) d t\right| \\
& \quad \leq \begin{cases}\text { const. }|u|_{E}|s||y-x|^{\alpha}, & |s| \leq 1, \\
\text { const. }|u|_{E}|y-x|^{\alpha}, & |s| \geq 1,\end{cases}
\end{aligned}
$$

and

$$
\left|\frac{1}{s+\varepsilon \int_{x}^{s+x} u}-\frac{1}{s+\varepsilon u(x) s}\right| \leq \begin{cases}\text { const. }|u|_{E}|s|^{\alpha-1}, & |s| \leq 1, \\ \text { const. }|u|_{E} s^{-2}, & |s| \geq 1,\end{cases}
$$

the first two terms in (A.12) may be bounded by

$$
\frac{\text { const. }}{1+x^{2}}|u|_{E}|w|_{E}|y-x|^{\alpha} \text {. }
$$


The final term in (A.12) may be integrated by parts to yield

$$
\frac{w(y)}{1+\varepsilon u(x)} \int_{0}^{\infty} s^{-1}\{u(x+s)-u(y+s)-u(x-s)+u(y-s)\} d s
$$

to which Privalov's theorem may be applied, and the result bounded by (A.14).

To estimate the third and last term in (A.11), we break the integral with respect to $s$ into ones over $(-1,1)$ and $(-\infty,-1) \cup(1, \infty)$. The use of our previous arguments yields the upper bound (A.14). Putting all of our estimates together yields

$$
|F(x)-F(y)| \leq \frac{\text { const. }}{1+x^{2}}|u|_{E}|w|_{E}|x-y|^{\alpha}, \quad|x-y| \leq 1,
$$

which with (A.10) proves the desired result (A.6).

\section{REFERENCES}

1. T. B. Benjamin, Internal waves of permanent form in fluids of great depth, J. Fluid Mech. 29 (1967), 559-592.

2. R. E. Davis and A. Acrivos, Solitary internal waves in deep fluid, J. Fluid Mech. 29 (1967), 593-607.

3. H. Ono, Algebraic solitary waves in stratified fluids, J. Phys. Soc. Japan 39 (1975), 10821091.

4. C. J. Amick and J. F. Toland, On solitary water waves of finite amplitude, Arch. Rational Mech. Anal. 76 (1981), 9-95.

5. $\ldots$, On periodic water waves and convergence to solitary waves in the long-wave limit, Philos. Trans. Roy. Soc. London A 303 (1981), 633-669.

6. __ Uniqueness and related analytic properties for the Benjamin-Ono equation-a nonlinear Neumann problem in the plane, Acta Math. 167 (1991), 107-126.

7. _ Uniqueness of Benjamin's solitary wave solution of the Benjamin-Ono equation, I.M.A. J. Appl. Math. 46 (1991), 21-28.

8. C. J. Amick and R. E. L. Turner, A global theory of internal solitary waves in two-fluid systems, Trans. Amer. Math. Soc. 298 (1986), 431-484.

9. __ Small internal waves in two-fluid systems, Arch. Rational Mech. Anal. 108 (1989), 111-139.

10. D. Gilbarg and N. S. Trudinger, Elliptic partial differential equations of second order, Springer-Verlag, 1977.

11. D. P. Bennett, R. W. Brown, S. E. Stansfield, J. D. Stroughair, and J. L. Bona, The stability of internal solitary waves, Math. Proc. Cambridge Philos. Soc. 94 (1983), 351-379.

12. N. K. Bary, A treatise on trigonometric series, Vols. I, II, Pergamon, 1964.

13. S. Agmon, A. Douglas and L. Nirenberg, Estimates near the boundary for solutions of elliptic partial differential equations satisfying general boundary conditions. II, Comm. Pure Appl. Math. 17 (1964), 35-92. 